ORIGINAL ARTICLE

\title{
The cognitive workload of air pistol shooters on the aiming task
}

\author{
TONGUÇ VARDAR ${ }^{1}$, FATIH SENDURAN² \\ ${ }^{1}$ Faculty of Physical Education and Sports Science, Uludag University, Turkey \\ ${ }^{2}$ Department of Physical Education and Sports, University of National Defense, Turkey \\ Correspondence to Dr. Fatih Senduran, Email. fsenduran@yahoo.com, Cell: +90 5062888240
}

\begin{abstract}
Background: Pupil dilations of the eye are known to correspond to central cognitive processes. Pupil size variations have been used for many years as a window on the mind, and various pupillary features have been associated with a number of cognitive processes

Aim: The aim of this study is to examine levels of cognitive workload that occur during the aiming task of air-pistol shooting athletes according to the change that takes place in the pupils.

Methods: Eight right-handed skilled male shooters (4 elite and 4 novice) were recruited in this study. A total of 320 shots - 160 dry (unscored) shots and 160 live (scored) shots - were recorded with a mobile eye-tracking device. The obtained pupil data were subjected to biometric analysis. The athletes' pupil sizes were determined with the independent samples t-test according to whether the shot made was scored or unscored and whether the athletes were elite or novice. The athletes' pupil size values at the beginning and end of the shot were obtained using the paired samples t-test.

Results: On completion of the shot, the sport shooters' pupils dilated on average by $2.85 \mathrm{~mm}$ at a rate of $32.5 \%$ compared to the situation at shot onset. Moreover, novice shooters' pupils dilated more when firing scored shots than when firing unscored shots. According to the research results, it can be said that sports shooters engage in intensive cognitive processes while aiming and trigger pulling immediately before firing the shot. Novice shooters engage in more intensive cognitive processes when firing scored shots than when firing unscored shots.

Conclusion: According to the results of the research, it can be said that in the sport of air pistol shooting, giving priority to dry (unscored) shooting training can contribute positively to the development of novice athletes' aiming task skills and cognitive load capacity.
\end{abstract}

Keywords: Cognitive workload, cognitive processes, eye tracking, air pistol, shooting

\section{INTRODUCTION}

While pistol shooting athletes are firing a shot under pressure, it is expected that for a successful shot, in the cognitive process immediately before pulling the trigger, their mental focus, visual attention, and trigger and breath control skills will be developed. For athletes to display successful performance, visual ability, focus and concentration of attention on the appropriate area are of critical importance.

The sport of pistol shooting is carried out by taking aim at a target 10 metres away. In this sport, the body and head do not move. The athlete's arm is raised towards the target, aim is taken, and the trigger is pulled at the correct time. This sport is more stationary than other branches of sport. However, a great deal more aiming and focusing ability is required. In air-pistol shooting, the period prior to the shot is crucial, as the shooter needs to prepare for the imminent shot release by pointing the pistol at the target and taking aim. ${ }^{2}$ Most importantly, the shooter must obtain an optimal sight picture, so that the position of the rear sight and fore-sight of the pistol are aligned correctly in relation to the target. ${ }^{3}$ In order to locate and maintain the aim of the pistol, the brain must process relevant visual information so as to position the pistol correctly in relation to the target. ${ }^{2}$ It seems reasonable to claim that both motor preparation and attentional processing are involved in proficient performance, such as that required in pistol shooting. ${ }^{4}$ In this study, air-pistol shooting performance was used as the motor task, since motor-cognitive processing while aiming is essential in proficient pistol shooting. ${ }^{5}$

Pupils are the black center of the eye. Their function is to let in light and focus it on the retina so you can see.
The size of the pupil is controlled by the activities of two muscles: the circumferential sphincter muscle found in the margin of the iris, innervated by the parasympathetic nervous system: and the iris dilator muscle, running radially from the iris root to the peripheral border of the sphincter. ${ }^{6}$ The normal pupil size in adults varies from 2 to $4 \mathrm{~mm}$ in diameter in bright light to 4 to $8 \mathrm{~mm}$ in the dark. The pupils are generally equal in size. They constrict to direct illumination (direct response) and to illumination of the opposite eye. ${ }^{6}$

Pupil size variations have been used for many years as a window on the mind, and various pupillary features have been associated with a number of cognitive processes. ${ }^{7}$ It is well known that pupil size reflects more than merely the amount of light that enters the eye. On the other hand, it was previously believed that pupil size was too simple a measure of brain activity. More recently, however, the discovery that activity in a part of the brain called the locus coeruleus causes changes in pupil size has offered the possibility that pupil size may be an important indicator of neural processing. ${ }^{8}$ This has resulted in the extensive use of pupil size as a secondary measure of cortical processing in psychology and neuroscience. ${ }^{9}$ It is known that pupil dilations of the eye correlate with central cognitive processes. A particular finding that has been made in this research is that those with higher cognitive ability have larger pupil sizes, even in a passive baseline condition. ${ }^{8}$

Eye-tracking technology is developing rapidly nowadays and light, wearable devices are now being produced. Eye-tracking technology is frequently used for eye monitoring and attention research studies. The development in the frequency ranges of mobile eye 
trackers has made it possible for researchers to also use pupil change data. However, although inherent to eyetracking algorithms, pupil size is estimated but rarely used for analysis. ${ }^{7}$

When the relationship between pupil size and cognitive load is examined in related studies in the literature, it is seen that the majority of these studies made by using an eye tracker are mostly concerned with areas such as problem solving (a mathematical operation, etc.), using the memory effectively and extensively, and reading skills. When the results of these studies are examined, it is seen that generally, as the intensity of the cognitive load increases and the difficulty of the problem needing to be solved increases, dilation of participants' pupils also occurs. In studies related to pupil size and cognitive load carried out in previous years, no study related to the sport of air-pistol shooting can be found.

When sports literature is examined, it is seen that in studies revealing the relationship between shooting sports and cognitive load, EEG systems are mostly used. EEG activities are mostly examined in these studies. Previous studies have demonstrated that successful air-pistol shooting performance can be distinguished from less successful performance by various EEG activities. Lose et al., ${ }^{2}$ for example, determined that successful performance in air-pistol shooting was preceded by considerably greater occipital alpha power prior to trigger pulls, while less successful performance was preceded by decreased occipital alpha power. Similarly, Del Percio et al., ${ }^{10}$ determined that compared to non-athletes, elite air-pistol shooters were characterised by greater cortical communication in the parietal and other posterior areas. Hoffing et al., ${ }^{7}$ investigated the cognitive processes affecting pupil size changes, and the reliability of these relationships, as a foundation for estimating how human cognitive processes and performance may vary in cognitively challenging, real-world tasks.

The use of Eye Tracking technology to study eye movements has been increased in the fields of academic and scientific research, market research, medical and human factors over the last period. ${ }^{11}$ Despite this fact that Eye Track technology is in many ways beneficial, it has been rarely used in the air pistol shooting task. Our study was designed to examine different levels of cognitive processes (according to pupil size) during dry (unscored) fire and live (scored) fire air-pistol shooting performances. We used pupillometry as this technique is the least invasive of existing measurements of neuro- and psychophysiological responses. ${ }^{12}$ Based on previous findings, we expected high activation of cognitive processes as reflected by pupil dilation.

\section{MATERIAL \& METHODS}

Sample: Eight right-handed skilled male shooters (4 elite and 4 novice) were recruited in this study. The athletes who took part in the research are licensed shooters of the Shooting and Hunting Federation of Turkey. To be defined as elite shooters, sportsmen/women were required to have at least 10 years' experience, to have taken part in national competitions, and to be currently active athletes. Elite shooters should also have participated in at least one international sports competition. Novice shooters were chosen from among shooting athletes with a maximum of 2 years' experience and athletes who had taken part in at least one national competition. They practised shooting regularly at least four times per week. The mean shooting score of the elite shooters was 575 and for the novice shooters, 545. The study was approved by an institutional review board, Uludag University, for the protection of the human subjects. All of the participants provided their informed consent.

\section{Instrument, Measures and Apparatus}

Air-pistol Shooting Task. In order to increase ecological validity, this study employed a real shooting task in accordance with normal contests rather than employing an electronic shooting training system. A $10 \mathrm{~m}$ range was assembled in a data collection building designed for the purpose, according to International Shooting Sport Federation regulations. The shooting task lasted for about 60 minutes per shooter.

Shooting Performance. Firstly, shooters were required to fire 20 dry (unscored) shots. After firing 20 dry shots, the shooters were asked to maintain their positions and not to leave the shooting range. Then, the athletes were asked to fire 20 live (scored) shots by squeezing the trigger. Therefore, the shooters each fired a total of 40 shots (20 dry and 20 live) during the training. No intervention was made in the athletes' shooting routines, timing, shooting techniques, experiences or shooting habits. The shooters did not see any researchers during shooting. The shooters were allowed to shoot under the same conditions as those in which they shot on a normal training day. Participants used their own pistols to carry out the shooting task so as to minimize unfamiliarity related to pistol handling. The bull's eye was awarded a score of 10 . The other eight concentric rings were each marked with different diameters (an increase of $0.8 \mathrm{~cm}$ per ring) and awarded different scores according to proximity to the bull's eye. The shot score and position for all participants were reported following each shot.

Pupil Movement Recording. As the hardware/technical device during data collection, an eye-tracking tool that analyses pupil movements, and can record data such as what the eye looks at and how long it remains fixed on a certain point, was used. The athletes' pupil movements while firing dry (unscored) and live (scored) shots were instantly recorded with the mobile eye-tracking device that they wore. To record the pupils with the eye-tracking tool, first of all the calibration process was carried out. Since each athlete's head structure and position of the eyes in the face were different, the calibration process was conducted separately for each shooter. Then, so that the athletes could become familiar with the device, they were asked to shoot freely for 5 minutes. For the athletes to feel comfortable and shoot at ease, the recording process was begun after they had entered the shooting range. In order to determine raw data showing where shooters looked in the real world, quiet eye duration and duration of focusing, the 'iMotions (2019)' computer software, ${ }^{13}$ which allows biometric measurements to be made, was used.

Procedures: The participants were requested not to consume drinks containing alcohol or caffeine for at least 24 hours before performing on the shooting range. Following the eye-tracking calibration procedure, the 
participants were requested to practise with ten warm-up shots. During the experimental recordings, all participants were requested to keep their body static and their eyes open for at least three seconds before shooting in order to minimize possible artifacts from body sway and blinking during shooting. For each shot fired by the athletes in their dry and live shot performances, their pupil sizes and changes, periods of focusing on the fore-sight, and quiet eye (QE) durations were determined with the eye-tracking device. The shooting athletes' pupil measurements began at QE onset and ended at QE offset. This process was carried out 40 times for each shooter, and so, a total of 320 times for all shooters. As a result of the analysis made, data for 160 dry shot performances and 160 live shot performances were obtained. The pupil movements carried out by the athletes while shooting were converted into digital data with the computer software algorithm. With this software, each performance realised by the shooting athletes (dry fire and live fire) was analysed one by one.

During the measurement of the athletes' performances, data that did not conform to the reliability values of the eye-tracking device and shots formed as a result of lost pupil data were not included in the study. The lights located in the shooting polygon were kept fixed throughout the research. Consequently, the amount of light had no effect on changes in the shooters' pupils.

Data Analysis: The distribution types of the obtained data were analysed with the Kolmogorov-Smirnov test of normality. The pupil sizes of the athletes who took part in the research were determined with the independent samples t-test according to whether the shot made was scored or unscored (live or dry) and also according to whether the athletes were elite or novice. The shooters' pupil size values at the beginning and end of the shot were obtained using the paired samples t-test. The level of statistical significance was set at $p=0.05$.

\section{RESULTS}

During assessment of the athletes, data that did not conform to the reliability values of the eye monitoring device were not included in the study. Accordingly, 126 dry (unscored) shots and 143 live (scored) shots of all athletes were included in the study. Therefore, a total of 269 scored and unscored shots of novice and elite athletes were used for determining the athletes' quiet eye durations.

Shooting Score Rates: The mean values of the scored shots fired by the novice and elite athletes during the research process are shown in Table 1.

Six zero values of shooting duration were excluded as missing data due to technical limitations of the eye tracker. No outliers were found. Hence, 143 valid cases out of 160 were analysed. A significant difference was found between shooting scores of novice and expert shooters ( $t=-$ 2.851, $p<.001)$. Expert athletes' shooting scores were higher than those of novice athletes $(9.37 \pm 0.80$ vs $9.72 \pm$ 0.63). Expert athletes' shooting scores were higher than those of novice athletes at a rate of $3.60 \%$.

Table 1: T-test Results Comparing Novice and Elite Athletes on

\section{Shooting Score}

\begin{tabular}{|l|l|l|l|l|l|l|}
\hline Athletes & $\mathrm{M}$ & $\mathrm{SD}$ & $\mathrm{M}$. Diff & $\mathrm{t}$ & $\mathrm{df}$ & $\mathrm{p}$ \\
\hline Novice & 9.37 & 0.80 & \multirow{2}{*}{0.35} & -2.851 & 141 & $\mathbf{0 . 0 0 * *}$ \\
\cline { 1 - 2 } Elite & 9.72 & 0.63 & & \\
\hline
\end{tabular}

${ }_{* \star}^{*} p<.001$

Pupil Diameter: Pupil diameter rates are presented in Table 2. During shooting performance, athletes' pupil sizes formed on completion of the shot were larger than their pupil sizes that existed at the start of the shot $(8.760 \pm$ 1.134 vs $5.910 \pm 2.17 \mathrm{~mm})(t=-23.737, p<.001)$. In other words, when the shot was completed, athletes' pupils dilated on average by $2.85 \mathrm{~mm}$ at a rate of $32.5 \%$ compared to the situation existing at the beginning of the shot (baseline).

Table 2: Descriptive Statistics, Paired and t-test Results on Onset and Offset Pupil Diameter of Athletes

\begin{tabular}{|c|c|c|c|c|c|c|c|c|c|}
\hline \multirow[t]{2}{*}{ Athletes } & \multirow[t]{2}{*}{$\begin{array}{l}\text { Shooting } \\
\text { Type }\end{array}$} & \multicolumn{2}{|c|}{$\begin{array}{l}\text { Onset } \\
(\mathrm{cm})\end{array}$} & \multicolumn{3}{|c|}{$\begin{array}{l}\text { Offset } \\
(\mathrm{cm})\end{array}$} & \multirow[t]{2}{*}{$\begin{array}{l}95 \% \mathrm{Cl} \\
\text { Mean Difference } \\
\end{array}$} & \multirow[b]{2}{*}{$t$} & \multirow[b]{2}{*}{$\mathrm{df}$} \\
\hline & & $\mathrm{M}$ & SD & $\mathrm{M}$ & SD & $\mathrm{n}$ & & & \\
\hline \multirow[t]{2}{*}{ Novice } & Dry Fire & 0.65 & 0.227 & 0.89 & 0.134 & 62 & $-0.23,0.219^{* a}$ & \multirow{2}{*}{$1,707^{* b}$} & \multirow[t]{2}{*}{134} \\
\hline & Live Fire & 0.60 & 0.257 & 0.90 & 0.087 & 74 & $-0.30,0.239^{* * a}$ & & \\
\hline \multirow[t]{2}{*}{ Elite } & Dry Fire & 0,54 & 0.198 & 0.84 & 0.111 & 64 & $-0.29,0.184^{* * a}$ & \multirow[t]{3}{*}{,$- 212^{b}$} & \multirow[t]{3}{*}{130} \\
\hline & Live Fire & 0.55 & 0.155 & 0.84 & 0104 & 68 & $-0.29,0.117^{* * a}$ & & \\
\hline Athletes & Total Shoot & 0.59 & 0.217 & 0.87 & 0.113 & 268 & $-0.28,0.104^{* * a}$ & & \\
\hline
\end{tabular}

${ }^{* *} p<.001,{ }^{*} p<.05,{ }^{a}$ paried test, ${ }^{b}$ t-test

According to shooting task type (dry fire and live fire) pupil diameter rates are presented in Table 3 and 4.

Table 3: Dry Fire(unscored) T-test Results of Athletes's Pupil Diameter

Differences (Offset-Onset)

\begin{tabular}{|l|l|l|l|l|l|l|}
\hline Athletes & $\mathrm{M}$ & $\mathrm{SD}$ & Mean Difference & $\mathrm{t}$ & $\mathrm{d}$ & $\mathrm{p}$ \\
\cline { 1 - 3 } Novice & $0.23 \mathrm{~cm}$ & 0.2 & -0.05 & -1.396 & \multirow{2}{*}{$0.0^{*}$} \\
\hline Elite & $0.29 \mathrm{~cm}$ & 0.1 & & & 12 \\
\hline
\end{tabular}

${ }^{*} p<0.05$

Table 4: Live Fire(scored) T-test Results of Athletes's Pupil Diameter Differences (Offset-Onset)

\begin{tabular}{|l|l|l|l|l|l|l|}
\hline Athletes & M & SD & Mean Difference & t & df & $\mathrm{p}$ \\
\hline Novice & $0.30 \mathrm{~cm}$ & 0.241 & -0.01 & 0.525 & 125 & 0.59 \\
\hline Elite & $0.29 \mathrm{~cm}$ & 0.118 & 0.59 & & \\
\hline
\end{tabular}

Novice Athletes: The paired dot plot presented in Figure 1

illustrates the relation onset and offset pupil diameter of

novice athletes. 
Figure 1: Pupil Diameter of Novice Athletes During Dry Fire and Live Fire

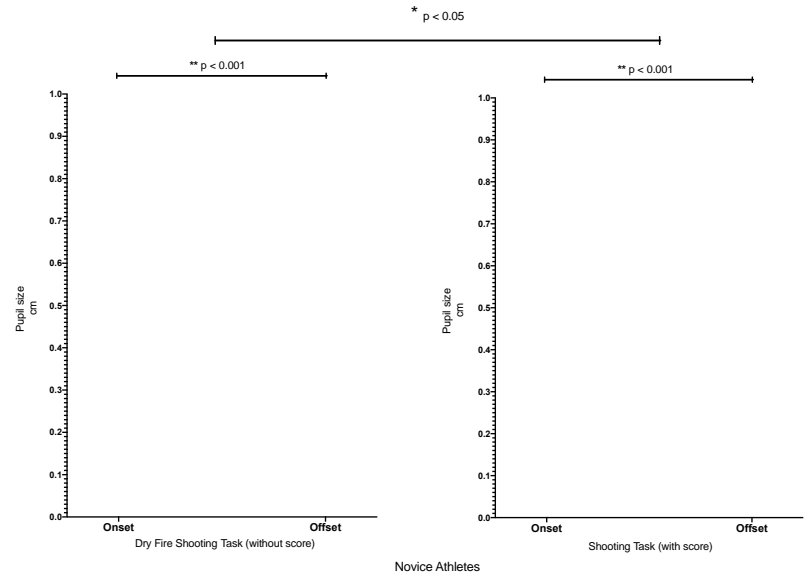

During dry fire (unscored) shooting, novice shooters' pupil sizes formed on completion of the shot were larger than their pupil sizes that existed at the beginning of the shot $(8.996 \pm 0.32$ vs $6.582 \pm 0.24 \mathrm{~mm})(\mathrm{t}=7.176, p<.001)$. In other words, during dry fire shooting, novice shooters' pupils dilated on average by $2.40 \mathrm{~mm}$ at a rate of $26.8 \%$ on completion of the shot. During scored (live) shooting, novice shooters' pupil sizes formed on completion of the shot were larger than their pupil sizes that existed at the beginning of the shot $(9.092 \pm 0.33$ vs $6.016 \pm 0.22 \mathrm{~mm})$ $(t=9.744, p<.001)$. In other words, during scored (live) shooting, novice shooters' pupils dilated on average by $3.08 \mathrm{~mm}$ at a rate of $33.8 \%$ on completion of the shot.

Elite Athletes: The paired dot plot presented in Figure 2 illustrates the relation onset and offset pupil diameter rates of elite athletes.

Figure 2: Pupil Diameter of Elite Athletes During Dry Fire and Live Fire

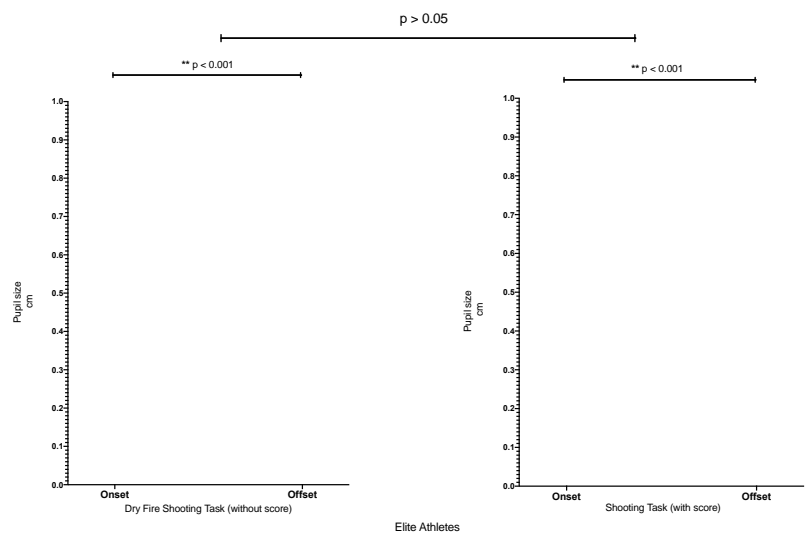

During dry fire (unscored) shooting, elite shooters' pupil sizes formed on completion of the shot were larger than their pupil sizes that existed at the beginning of the shot $(8.447 \pm 0.329$ vs $5.483 \pm 0.23 \mathrm{~mm})(t=10.37, p<.001)$. In other words, during dry fire shooting, elite shooters' pupils dilated on average by $2.95 \mathrm{~mm}$ at a rate of $35.8 \%$ on completion of the shot. During scored (live) shooting, elite shooters' pupil sizes formed on completion of the shot were larger than their pupil sizes that existed at the beginning of the shot $(8.484 \pm 0.29$ vs $5.582 \pm 0.24 \mathrm{~mm})(t=12.76$, $p<.001)$. In other words, during scored (real) shooting, elite shooters' pupils dilated on average by $2.90 \mathrm{~mm}$ at a rate of $34.2 \%$ on completion of the shot.

Pupil Diameter Differences Between Onset and Offset: The bar graph presented in Figure 3 illustrates the relation onset and offset pupil diameter differences rates of athletes during dry and live fire shooting tasks. When the changes in novice athletes' pupil sizes that occurred during dry fire shooting are compared with the changes in their pupil sizes that occurred during scored shooting are compared $(2.40 \pm$ 0.21 vs $3.08 \pm 0.23 \mathrm{~mm}$ ), it is seen that novice shooters' pupils were more dilated when firing scored shots than when firing dry shots $(t=1.693, p<.05)$.

When the changes in elite athletes' pupil diameter that occurred during dry fire shooting are compared with the changes in their pupil diameter that occurred during scored shooting $(2.95 \pm 0.18$ vs $2.90 \pm 0.11 \mathrm{~mm})$, it is seen that dilation rates of elite shooters' pupil sizes for scored and unscored shots were very similar to each other ( $t=-$ $0.212, p=0.83$ ).

Figure 3: Pupil Diameter Differences (Offset-Onset) of Athletes During Dry Fire and Live Fire

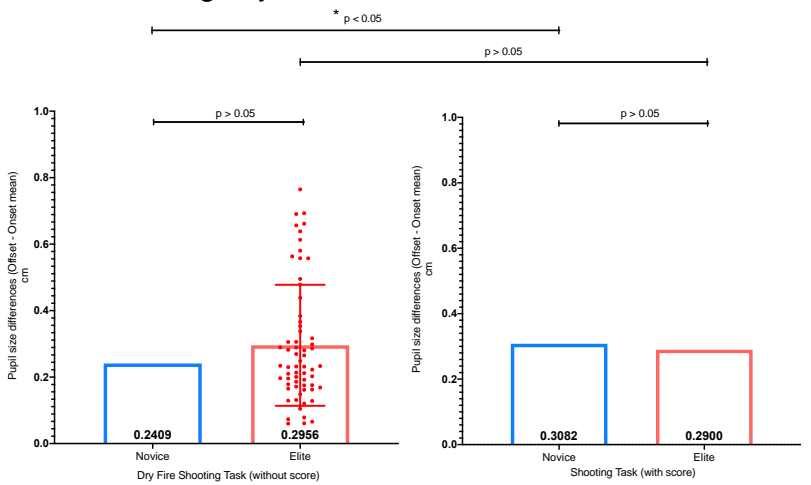

\section{DISCUSSION}

The aim of this study was to examine levels of cognitive load that occur during shooting performance of pistol shooting athletes by considering the change that takes place in the pupils. One of the main reasons for conducting this study with pistol shooters was that this sport can be performed with minimal movement. It is known that just like sudden changes of light, exercise also affects pupil dilation. Ishigaki et al., ${ }^{14}$ reported that the pupil dilates during exercise, while during the period after completion of exercise, contraction of the pupil occurs. The diameter of the pupil reaches its highest level under maximum exercise conditions. In exercises done under a constant load, however, the diameter of the pupil increases as the period of exercise is extended. ${ }^{14}$ In this study, the lights in the polygon where the athletes fired shots were kept fixed. Moreover, the shooting athletes did not do any exercise activities. Therefore, during the pistol shooters' performances, no situation occurred that would externally affect the dilation of their pupils. A number of variables affect success in the sport of shooting. Shooting success is affected by numerous interrelated factors such as arrangement of correct stance and centre of balance, applying the principles of aiming, trigger and breath control, 
period of focusing on target, etc. In the air-pistol shooting branch of the sport, which includes an extremely complex psychomotor performance process, a shooter's process of entering the line of sight, pointing his weapon at the target and taking aim are the important steps that need to be taken correctly before firing the shot. During this process, the shooter must adopt the correct stance and capture the optimal image, known as the sight picture (optimal alignment of rear sight and foresight). ${ }^{15}$ As expected, in this study, elite shooters achieved higher shooting scores than amateur shooters. Considering the training history and experience in national and international competitions of elite shooting athletes, their higher shooting scores than those of novice shooters can be regarded as an expected result.

The conducted studies reveal that the baseline diameter of the pupils, which begin to dilate with the cognitive process, are independent of each other. ${ }^{16}$ For this reason, comparison of baseline pupil diameters in athletes who took part in the research was not included in the study, and to obtain the findings, the differences between the shooters' baseline and final pupil diameters were considered. Increases in brain activity that occur in different regions of the human brain can directly cause dilation of the pupil. Thus, it can be possible to understand the cognitive processes in depth with the data obtained. ${ }^{17}$ One reflection of the processes occurring in the central brain results in changes in pupil diameter and movement of the pupil. $8,18,21$

When the pupil diameter of amateur shooting athletes in this study was examined according to type of shot fired, amateur shooters' pupils dilated at a rate of $26.8 \%$ for unscored shots and $33.8 \%$ for scored shots. It was seen that the novice shooters' pupil sizes varied according to whether the shot fired was scored or unscored. When amateur shooters fired scored shots, their pupils dilated by $0.68 \pm 0.11 \mathrm{~mm}$ more than when they fired unscored shots. While amateur shooting athletes' pupils dilated by $3.08 \pm$ $0.23 \mathrm{~mm}$ when firing scored shots compared to their size at the beginning of shots, they dilated by $2.40 \pm 0.21 \mathrm{~mm}$ when firing unscored shots. John et al., ${ }^{16}$ reported that small changes of less than $0.5 \mathrm{~mm}$ occurring in the pupil are each a small reflection of brain activation occurring in the human cognitive process. In this study, the fact that amateur athletes' pupils dilated by $0.68 \mathrm{~mm}$ on average may indicate that brain activity occurring in the cognitive process was more intense. Since amateur shooters had no numerical proof of the shot when firing an unscored shot, it may be considered that they concentrated less and did not focus accurately enough on the fore-sight. While firing a scored shot, however, it can be said that amateur shooters paid more attention and experienced more cognitive load.

When the pupil dilation of elite shooting athletes in this study is examined according to type of shot fired, elite shooters' pupils dilated at a rate of $35.8 \%$ for unscored shots and $34.2 \%$ for scored shots. The obtained findings reveal that the rates of change in elite shooters' pupil diameter between start of shot and end of shot for scored and unscored shots were very similar to each other. This situation can be said to be a benefit of the years of training conducted by elite shooters and the championships they have participated in. In other words, the shooting discipline, focusing, and sight control displayed and the cognitive load experienced during shooting performance by elite shooting athletes does not change according to whether the shot is scored or unscored. While examining the cognitive processes that occur in the period immediately before making the shot in sports in which a target is fired at (archery, darts, etc.) like air-pistol shooting, researchers have used the electroencephalogram (EEG) to measure brain activity. ${ }^{2}$ In these studies, it is reported that the EEG alpha power occurring in the left anterior-temporal lobe of the brain is considerably greater than that occurring in the right lobe. In the research by Tremayne and Barry, ${ }^{4}$ examining physiological activity models in elite and novice pistol shooters, it was determined that in elite shooters, there was a slow reduction in heart rate levels and skin conductance before the shot, and that there was a 'rebound' increase immediately after the shot, characteristics which were not observed in novice shooters.

It is known nowadays that the dilation and contraction of the pupil is related to the central cognitive processes. ${ }^{8}$ Pupil size can be used as an indicator of instantaneous changes in stimulation, mental effort and attention. ${ }^{19,20,8}$ Tsukahara et al., ${ }^{8}$ stated that when fluid intelligence is in question, that is, when "reasoning and problem-solving skills in original and new situations" are required, the working capacity of the memory is correlated with pupil diameter. In another study made on cognitive load, it was revealed that dilation occurring in the pupil increased in proportion to the difficulty of the mathematical problem that the subject was attempting to solve. ${ }^{16}$

Considering the studies conducted in the literature, it is seen that an increase occurring in pupil size corresponds with an increase in the cognitive process. A greater effort by the human brain to process emotion or information results in greater pupil diameter. In the shots fired by pistol shooting athletes participating in this study, a mean increase of $32.5 \%$ in athletes' pupil diameter occurred between shot onset and shot completion. The shooters' pupil diameter increased significantly at $Q E$ offset compared to QE onset.

\section{CONCLUSION}

In conclusion, during the shooting athletes' performances, the fact that their pupil diameter at the beginning of the shot had increased by the end of the shot can be expressed as an indicator that the athletes experienced a significant cognitive load during this process. Moreover, this dilation that occurred in the pupils indicates that the athletes thought intensely about the actions they had to carry out (holding, aiming, trigger pulling, etc.) in order to form a correct sight picture and to carry out a successful shot. Moreover, similar dilations of an athlete's pupils in both scored and unscored shots during shooting performance may indicate that the shooter is at the elite athlete stage. According to the findings obtained in our study and to studies made in the literature, one can say that conducting special exercises especially dry (unscored) shooting training, will make a positive contribution to developing novice athletes' performance, cognitive load capacity and aiming shooting skill in the process of becoming elite athletes. 


\section{RECOMMENDATIONS}

This study was carried out only on pistol shooting athletes. It is considered that inclusion of rifle shooting athletes would be appropriate in future studies.

In future studies to be made with shooting athletes, it is considered that the use of an eye-tracking system and other research instruments (e.g., EEG, EMG, HRV, etc.) together in order to analyse different processes (mental perception, visual perception, coordination, etc.) in athletes' performance will contribute to obtaining more comprehensive and sensitive results.

\section{Disclaimer: None.}

Conflict of interest: None.

Source of funding: None

\section{REFERENCES}

1. Vickers J. N. Origins and current issues in Quiet Eye research. Current Issues in Sport 2016.

2. Loze G. M., Collins, D., Holmes, P. S. Pre-shot EEG alphapower reactivity during expert air-pistol shooting: $A$ comparison of best and worst shots. Journal of Sports Sciences 2010; 19(9), 727-733.

3. Leatherdale F., Leatherdale, P. Successful Pistol Shooting. Crowood Press 1995.

4. Tremayne P., Barry, R. J. Elite pistol shooters: Physiological patterning of best vs. worst shots. International Journal of Psychophysiology 2001; 41(1), 19-29.

5. Cheng M. Y., Wang K. P., Hung C. L., Tu, Y. L., Huang, C. J., Koester, D., Hung, T. M. Higher power of sensorimotor rhythm is associated with better performance in skilled airpistol shooters. Psychology of Sport and Exercise 2017; 32, 47-53.

6. Walker H., Hall W.D., Hurst J.W. The Pupils. Clinical Methods: The History, Physical, and Laboratory Examinations 1990. 3rd edition. Butterworths.

7. Hoffing R. A. C., Lauharatanahirun N., Forster D. E., Garcia, J. O., Vettel J. M., Thurman S. M. Dissociable mappings of tonic and phasic pupillary features onto cognitive processes involved in mental arithmetic. PLOS ONE 2020; 15(3), 1-14.

8. Tsukahara J. S., Harrison T. L., Engle R. W. The relationship between baseline pupil size and intelligence. Cognitive Psychology 2016; 91, 109-123.
9. Ebitz R,B., Moore T. Both a Gauge and a Filter: Cognitive Modulations of Pupil Size. Front Neurol 2019; 9:1190.

10. Del Percio C., lacoboni M., Lizio R., Marzano N., Infarinato, F., Vecchio, F., Babiloni, C. Functional coupling of parietal alpha rhythms is enhanced in athletes before visuomotor performance: A coherence electro encephalographic study. Neuroscience 2011; 175, 198-211.

11. Skvarekova I., Skultety, F. (2019). Objective measurement of pilot's attention using eye track technology during IFR flights. Transportation Research Procedia 2019; 40: 15551562.

12. Peysakhovich V., Dehais F., Causse M. Pupil Diameter as a Measure of Cognitive Load during Auditory-visual Interference in a Simple Piloting Task. Procedia Manufacturing 2015; 5199-5205.

13. iMotions. iMotions Biometric Research Platform 2019.

14. Ishigaki H., Miyao M., Ishihara S. Change of pupil size as a function of exercise. Journal of Human Ergology 1991; 20, 61-66.

15. Hawkins, R. Identifying mechanic measures that best predict air-pistol shooting performance. International Journal of Performance Analysis in Sport 2011; 11(3): 499-509.

16. John T. Cacioppo, Louis G. Tassinary, Gary G. Berntson (Eds.), Handbook of Psychophysiology, 2nd. Cambridge University Press 2000.

17. Siegle G. J., Steinhauer S. R., Stenger V. A., Konecky, R., Carter, C. S. Use of concurrent pupil dilation assessment to inform interpretation and analysis of fMRI data. Neurolmage 2013; 20(1), 114-124.

18. Beatty J. Task-evoked pupillary responses, processing load, and the structure of processing resources. Psychological Bulletin 1982; 91(2), 276-292.

19. Beatty, J., \& Lucero-Wagoner, B. (2000). The pupillary system. In J. T. Cacioppo, L. G. Tassinary, \& G. G. Berntson (Eds.), Handbook of psychophysiology, Cambridge University Press 2000; (2nd ed.,pp. 142-162).

20. Hess E. H., Polt J. M. Pupil size as related to interest value of visual stimuli. Science 1960; 132(3423), 349-350.

21. Ozdemir, M., Tanir, H., Ilkim, M., \& Ozmaden, M. (2017). The effects of 8 week exercise program on reaction time performance of hearing impaired students at 11-14 years of age. 